\title{
Design of Auto-path-tracking Car based on Photoelectric Sensor
}

\author{
Zhou Hao ${ }^{1, ~ a ~ a n d ~ L i u ~ X i a o j u n ~ 2, b ~ * ~}$ \\ ${ }^{1}$ School of Transportation, Huanggang Normal University, Hubei Huanggang, China \\ ${ }^{2}$ School of Transportation, Huanggang Normal University, Hubei Huanggang, China \\ a18623582@qq.com mail, bwhutliuxiaojun@126.com \\ * The corresponding author
}

Keywords: Photoelectric Sensor; Smart Car; Auto-path-tracking; Path Recognition

\begin{abstract}
The paper introduces the design of the Auto-path-tracking Car. The software and hardware design method which realizes the autonomous tracing using the infrared reflective photoelectric sensors as the path recognition module is researched. The system employs Freescale HCS series 16 bit single-chip microcomputer MC9S12XS128 as its main controller and an array of photoelectric sensors for recognizing the path information. S128 energizes the servor to steer and control the speed of the DC electric motor according to the analysis of the path and speed information that from sensors. Consequently this intelligent car can track the black-guide-line automatically and move forward following the line quickly and smoothly.
\end{abstract}

\section{Introduction}

With the extensive application of intelligent transportation system, the control of smart car system has become a concern area, which covers a wide range of knowledge, including artificial intelligence, computer control, sensor path recognition and other knowledge[1]. The design of "Freescale" smart car game as the background, using Freescale's 32-bit microcontroller MC9S12XS128 as the core controller, the use of a laser sensor acquisition path information, designed a self-tracking intelligent car automatic control system, has the characteristics of simple structure, strong processing power, good robustness and so on[2-4].

\section{Intelligent Car Hardware Design}

The overall structure of the system. The overall workflow of the smart car control system is: the photoelectric sensors for recognizing the path information, passes the signal into the XS128 single chip computer, filters the collected image through the image signal processing algorithm, and carries on the software binary value to the filtered image And then further processing to obtain the main track information; through the photoelectric encoder to detect the speed, through the XS128 input capture interrupt pulse calculation, and get the current speed of the car; the processed data after processing into the path recognition algorithm module, To obtain control rules, in accordance with the rules of control of the steering gear steering gear and motor speed for accurate and rapid control. The smart car control system studied in this paper adopts the PID control algorithm to control the motor and the steering engine ${ }^{[5]}$. Smart car control system structure shown in Fig.1. In order to make the whole system simple and efficient, in the design process as much as possible to optimize the existing hardware structure, thereby reducing the hardware problems caused by the failure of the entire control system.

XS128 single chip minimum system module. The control chip used in this paper is FREESCALE MC9S12XS128 (referred to as XS128) series, which is a 16-bit microcontroller, floating point operation, FLASH 128K, the paper designed XS128 minimum system board with $150 \mathrm{M}$ standard frequency, through the test lock Phase ring up to $400 \mathrm{M}$, the bus frequency up to $200 \mathrm{M}$, can make the entire control system the best, the most stable play. At the same time XS128 microcontroller also has the characteristics of low power consumption, reducing the overall system power supply burden. XS128 minimum system includes 3.3V power supply, reset circuit, crystal oscillator circuit and I/O 
port and other components [6-7]. The minimum system is necessary for the operation of the microcontroller, and the formation of the smallest system will not change, the design will XS128 minimum system made of a separate module, easy to use and replace.

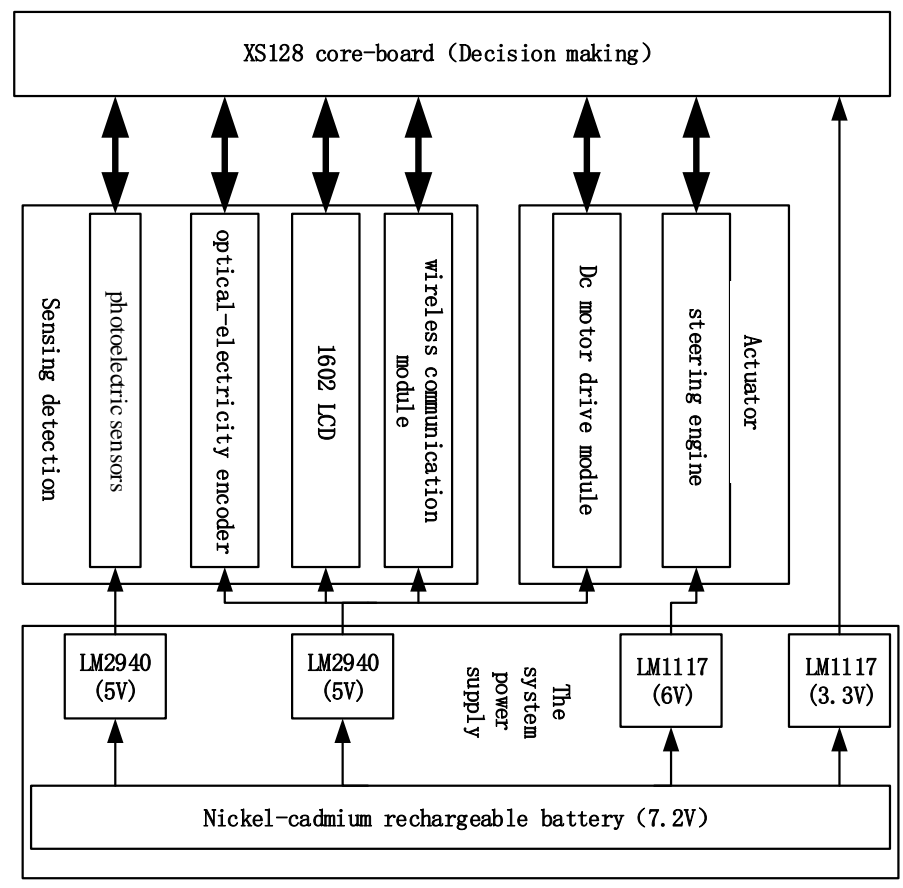

Figure 1. The block diagram of system

Power regulator module. In the entire smart car control system, the system consists of a $7.2 \mathrm{~V}$ nickel-cadmium rechargeable battery to provide power in the entire model vehicle system, but because the different modules need different voltage, in order to make these modules are able to work smoothly, The $7.2 \mathrm{~V}$ voltage of the battery is regulated to the operating voltage of these modules, respectively. In the design of the regulator module, not only to consider the size of the regulator and the current capacity and other basic parameters, but also pay attention to voltage conversion ratio, noise handling, circuit protection and electrostatic shielding and other factors. The voltage regulator used in this paper is the $5 \mathrm{~V}$ regulator chip LM2940 and $3.3 \mathrm{~V}$ regulator chip LM1117. Stable regulator effect is the basis for intelligent control, but also the premise of the stability of the entire system. The power supply relationship of each module is shown in Fig.2.

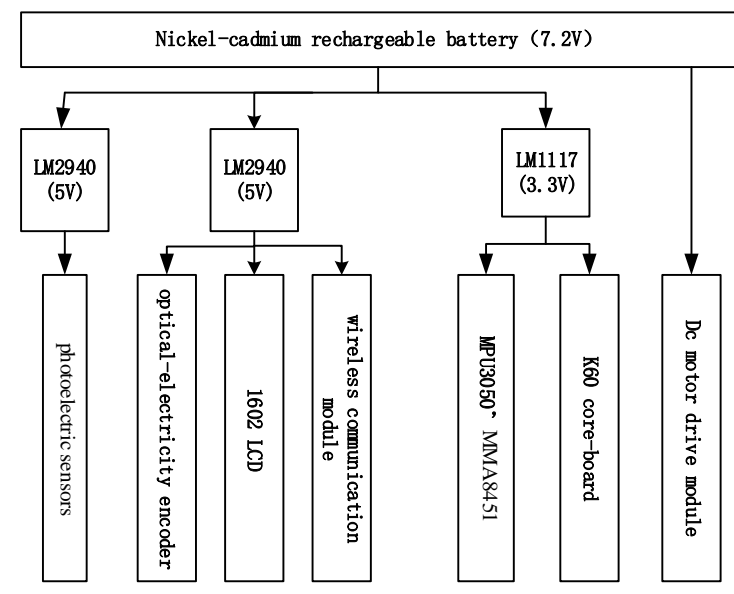

Figure 2. Power supply diagram

Execution module. XS128 completes the information collection, and after the algorithm processing, completes the decision-making, outputs the control signal. At this time by the implementation of the module to achieve smart car speed and steering control. The execution module 
is shown in Fig.4, including the steering gear and the motor and its drive circuit. The steering gear S-D5 digital servo is controlled by a three - wire control, in which the control line is connected to the XS128 pin PTA10 and is turned according to its output PWM signal. Motor drive chip selection of two BTS7960, using $\mathrm{H}$ bridge drive, in order to achieve rapid speed adjustment.

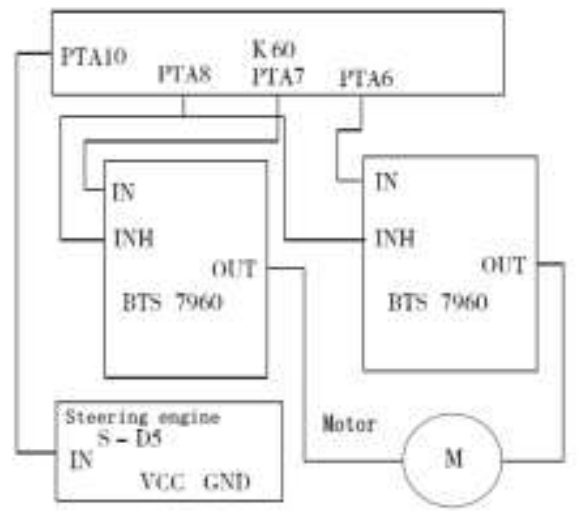

Figure 3. Hardware circuit diagram of the execute module

Path detection module. As the infrared photoelectric sensor is cheap, the circuit design is simple, it is often used. The RPR220 is an all-in-one reflective photodetector that recognizes the direction of the track by recognizing two colors that are significantly different in reflectance, such as black and white. The design uses a total of eight RPR220-type infrared sensors, the level of uniform in front of the car racing sensor board, due to its prospective and poor, usually only $3 \mathrm{~cm} \sim 5 \mathrm{~cm}$, so the sensor board hanging in front of the car, the use of vertical detection method. The sensor pitch is $12 \mathrm{~mm}$, less than the black line width of the track, ensuring that the sensor always detects the black line when the car is driving on the track. The 8 sensors of the car can detect 8 precise positions, plus two adjacent sensors detect the black line at the same time and no sensor detects the black line. There are 16 kinds of detection states in total, so the lateral detection accuracy can reach $6 \mathrm{~mm}$, basically meet the tracing requirements.

Speed detection module. The main function of the tachometer module hardware is to send the analog signal of the frequency change with the speed to the signal processing circuit and finally to the digital pulse signal. In order to precisely control the movement of the car, we use the method of single-chip control encoder to detect the motor speed of the car. we use a 100-line rotary encoders OME-100-1N optical encoder produced by OMRON company, with a 1: 1 gear ratio with a pair of gear connected to the drive shaft, the drive shaft rotates a week, the encoder can get $100 \mathrm{~A}$ pulse, the microcontroller through the pulse count can get the specific value of speed.

\section{Design and Implementation of Software System}

General design of software system. The main program is only responsible for initialization and data transmission, LCD display and other tasks, in the timer interrupt in the sensor data acquisition, control the amount of control and control output and other operations. The main functions of the software include:(1) the sensor signal acquisition, processing; (2) motor PWM output; (3) car mold operation control: upright control, speed control, direction control; (4) car mold operation process control: program initialization, car mode start and end, Car model status monitoring; (5) car model information display and parameter settings: status display, host computer monitoring, parameter settings, etc. The first class includes 1-3 functions, which belong to the need for precise time period execution, and thus can be done in a periodic timed interrupt. The second category includes the 4-5 function. Its execution does not require an accurate time period. System software flow chart shown in Fig.4. 


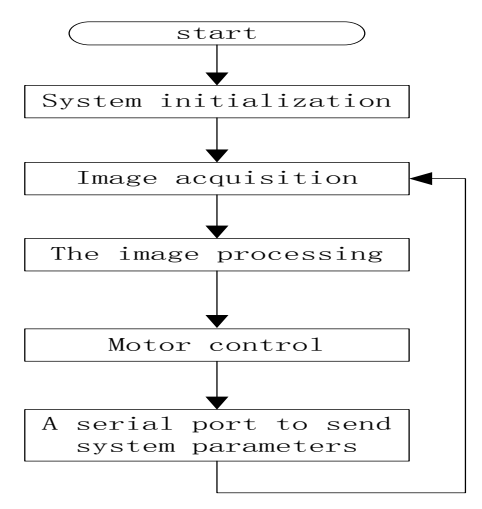

Figure 4. System software flow pattern

Path recognition algorithm. During the autonomous vehicle tracing process, the photoelectric sensor may be disturbed by environmental factors such as external light, vehicle body jitter, cross line, up-down slope and path dark spot, which may affect the stability of the tracing of the vehicle due to the deviation of the detected path information. To this end, we use a continuous detection filter to eliminate interference: the sensor detects five consecutive paths and stores the collected information in a two-dimensional arrayLine $[i][n]$. Check the black line value 1 , otherwise save the value 0 . Otherwise stored value 0 .

If $\sum_{i=1}^{i=5} \operatorname{Line}[i][n] \geq 3$, the black line is detected in the nth column of Line $[i][n]$, line $[n]=1$; otherwise it is interference, line $[n]=1$, For the array line[8]. Since the sensor spacing is slightly less than the black line width, line [8] typically has 16 states: extra two for 1 (path crossing detected), one or two for 1 (black line detected), all 0 (No black line detected).

Steering control. According to the degree of deviation from the central location of the car to the car to allocate different sizes of steering value, the basic idea is the greater the degree of deviation to the car the greater the value of the steering, which can ensure that the deviation from the track center in the case of the car to the maximum steering value to ensure that the car quickly back to the center position, the algorithm is used in the PD control: steering PWM value calculation formula:

$$
P W M=K p e(t)+K d[e(t)-2 e(t-1)+e(T-2)]
$$

Simple PD control in the car in the low-speed state when the effect can be, with the speed of the car in the steering angle of the angular speed should be increased accordingly, in the face of continuous steering, due to inertia, steering time is serious Delayed the phenomenon, causing the car out of the track. In order to solve this problem, the system also measured the angular velocity of the normal rotation around the track plane. Similarly, the PD algorithm calculates a gain to offset the steering delay due to inertia [7-9]. By constantly adjusting the size of the PD value and observe the phenomenon, to obtain the appropriate PD value, the more perfect solution to the problem of steering delay.

Speed control. Using the pulse capture port on the microcontroller to capture the vehicle speed sensor in the unit time pulse to obtain the current speed, PI closed-loop control, according to the actual load conditions and rapid adjustment of the PWM wave to adjust the torque in time, and ultimately stabilize the car And in order to avoid the interference of the speed control to the upright control, the sum of the 10 main cycle periods is measured when measuring the motor speed, and the output of the PI control is divided by 10 and the output is 10 times. The incremental PID calculation The formula is:

$$
\operatorname{Kpe}(\mathrm{t})+\mathrm{Ki}[\mathrm{e}(\mathrm{t})-\mathrm{e}(\mathrm{t}-1)]
$$

In the ordinary track, PI control is sufficient to meet the requirements, but in the passage of about $10^{\circ}$ ramp, the simple PI control cannot produce enough acceleration car, and downhill cannot decelerate in time, which is not conducive to fast and smooth Complete the game. In order to solve this problem, in the car uphill to improve the zero deviation of the accelerometer, so that it has a 
tendency to tilt forward, and downhill to reduce the zero deviation of the accelerometer, so that it has a tendency to tilt backward Produce enough acceleration to accelerate and decelerate. In addition, on the ordinary track, the speed deviation of the car is also limited, more than the limit is to increase or decrease the zero deviation of the accelerometer, so that the corresponding direction of tilt, resulting in an additional acceleration.

\section{Conclusion}

In this paper, a design method of intelligent vehicle system based on infrared photoelectric sensor tracing is proposed. The system uses MC9S12XS128 microcontroller as the main controller and DC motor as the executive components. The key design and analysis of the intelligent vehicle is proposed. The elimination of continuous detection filter is proposed Interference mode. By programming the module used to initialize the first, and through the corresponding data register or status register read and write to achieve the desired function. The test results show that the smart car can achieve high speed and stability on the straight road, control the speed on the curve, and the smart car can run smoothly.

\section{Acknowledgements}

The project was supported by the project of Huanggang Normal University (Grant No. zxfz2016A014).

\section{References}

[1] MILLER R M, SHAH S L, WOOD R K, etal. Forecast and PID control technology [J]. ISA Transactions, 2008,38(1):11-23.

[2] NORMEY-RICOJE, GOJ, CAMACHOEF. A Smith-predictor-based generalized predictive controller for mobile robile path-tracking [J].Control Engineering Practice, 2008, 16(4):407-428.

[3] GAO Zheng-zhong, ZHAO Li-na, LI Shi-guang, BAI Xing-zhen, SONG Sen-sen. Design of Intelligent Vehicle Control System Based on Camera[J]. Automation \& Instrumentation(Chinese), 2015, 32(6), 1-5.

[4] Yang Zhengcai, Lü Ke, Zhu Le. Design of Upright - moving Intelligent vehicle Based on 32bit Kinetis - K60 Microcontroller of Freescale[J]. Journal of Hubei University of Automotive Technology(Chinese), 2014, 28(2), 46-51.

[5] XU Wei, CHEN Wanmi.Design of a Predictive Adjustable Two Wheel Upright Intelligent Car[J]. Industrial control computer(Chinese), 2016, 29(8), 45-49.

[6] Peng L. Individual Vision and Peak Distribution in Collective Actions [J]. Communications in Nonlinear Science and Numerical Simulation. 2017, 47: 238-252.

[7] QIN Gang, DU Chao, WU Danyi. Simulation and Analysis on Intelligent CarTurning Control Algorithm[J]. Techniques of Automation \& Applications(Chinese), 2012, 31(12), 49-64.

[8] YU Shaodong, HUANG Danping, TIAN Jianping, HU Yong. Control System Design of Intelligent car Based on Kinetis K60 [J]. Journal of Sichuan University of Science \& Engineering(Natural Science Edition,Chinese), 2014, 27(5), 37-42

[9] GAO Zheng-zhong, ZHAO Li-na, LI Shi-guang, BAI Xing-zhen, SONG Sen-sen. Design of Intelligent car Control System Based on Camera[J]. Automation \& Instrumentation(Chinese), 2015, 32(6), 1-5

[10] Yang Zhengcai, Lü Ke, Zhu Le. Design of Upright-moving Intelligent car Based on 32-bit Kinetis - K60 Microcontroller of Freescale[J]. Journal of Hubei University of Automotive Technology(Chinese), 2014, 28(2), 46-51 